\title{
INFERTILIDAD FEMENINA ASOCIADA A INFECCIÓN POR CHLAMYDIA TRACHOMATIS
}

\author{
FEMALE INFERTILITY RELATED TO AN INFECTION CAUSED BY CHLAMYDIA \\ TRACHOMATIS
}

\author{
INFERTILIDADE FEMININA ASSOCIADA A INFEÇÃO PELA CHLAMYDIA TRACHOMATIS
}

\section{GUILLERMO BEJARANO WAGNER', WILLIAM HURTADO RÍOS², DIEGO VÁSQUEZ CEDEÑO'}

\author{
1 Universidad Católica de Santiago de Guayaquil, Guayaquil, Ecuador \\ ${ }^{2}$ Hospital Teodoro Maldonado Carbo, Guayaquil, Ecuador
}

\begin{abstract}
Resumen
Antecedentes: la infección por Chlamydia trachomatis es la enfermedad de transmisión sexual más frecuente en todo el mundo y es una causa importante de infertilidad en mujeres debido a que produce obstrucción de las trompas de Falopio. Tratar esta infección a tiempo reduciría la infertilidad y el daño que produce en el tracto reproductor femenino. Objetivos: determinar la frecuencia de infección por C. trachomatis en mujeres infértiles y su asociación con los diferentes tipos de infertilidad, los antecedentes de abortos, su pronóstico reproductivo y las alteraciones en el espermatograma de la pareja sexual. Método: se realizó un estudio transversal en el hospital Teodoro Maldonado Carbo de la ciudad de Guayaquil, donde se evaluó la frecuencia de infección por $C$. trachomatis en 101 mujeres en edad reproductiva con deseos de fertilidad durante los años 2012 y 2013. Se realizó la detección de anticuerpos IgM e lgG por ELISA, y se asoció su presencia con las distintas variables del estudio. Resultados: el 65,35\% de las mujeres estudiadas presentaron anticuerpos positivos para C. trachomatis. La asociación de la infección con las variables no fue estadísticamente significativa, con la excepción de las alteraciones en el espermatograma de la pareja sexual. Conclusión: la infección por C. trachomatis en mujeres infértiles es alta. Se recomienda implementar en Ecuador un monitoreo de rutina para diagnosticar y tratar la infección de manera oportuna y evitar que se vuelva crónica.
\end{abstract}

PALABRAS CLAVE: Chlamydia Trachomatis, infertilidad.

Abstract

Background: infections by Chlamydia trachomatis are the most frequent sexual transmission diseases worldwide and they are an important cause of infertility in women since it produces obstruction in the Fallopian tubes. Early treatment of this disease will reduce infertility rates and damage produced in the Female Reproductive Tract. Objective: to determine the frequency of infection by C. trachomatis in infertile females and its association with other types of infertility, rates of abortions, reproductive prognosis, and alterations in the spermogram of the sexual partner. Method: a cross-sectional study was carried out at the Teodoro Maldonado Carbo hospital in Guayaquil city. From 2012 to 2013, frequency of infection by C. trachomatis was assessed in 101 women at their reproductive age and desiring pregnancy. Detection of $\operatorname{lgM}$ and $\lg G$ antibodies was performed by ELISA, and the presence of the different variables under study were associated. Results: $65 / 35 \%$ of the women under study showed positive antibodies to $C$. trachomatis. The association of the infection with the variables was not statistically significant, except for the alterations in the spermogram of the sexual partner. Conclusion: infection by C. trachomatis in infertile women is high. It is advisable to implement routine monitoring to diagnose and to treat the infection in an effective way and to prevent it from becoming a chronic disease

KEYWORDS: Chlamydia trachomatis, infertility.

Resumo

ANTECEDENTES: a infeção pela chlamydia trachomatis é doença de transmissão sexual mais frequente em tudo mundo, e é uma causa importante de infertilidade em mulheres devido a que produz obstrução das trompas de Falópio. Curar esta infeção a tempo reduziria a infertilidade e o dano que se produz no trato reprodutor feminino. Objetivos: determinar a frequência de infeção por c. Trachomatis em mulheres férteis e a sua associação com os diferentes tipos de infertilidade, os antecedentes de abortos, ou seu prognostico reprodutivo e as alterações no espermograma do casal sexual. Método: um estúdio transversal foi feito no hospital Teodoro Maldonado Carbo da cidade de Guayaquil, onde a frequência de infeção pela c. Trachomatis foi avaliada em 101 mulheres em idade reprodutiva com desejo de fertilidade durante os anos 2012 e 2013. A deteção de anticorpos $\lg M$ e lgG pelo método ELISA foi realizado e a sua presencia foi associada com as distintas variáveis do estudo. Resultados: 0 65,35\% das mulheres estudadas apresentaram anticorpos positivos para C. Trachomatis. A associação da infeção com as variáveis não foi estadisticamente significativa, com a exceção das alterações no espermograma do casal sexual. Conclusão: a infeção pela $C$. Trachomatis em mulheres férteis é alta. E recomendado implementar no Equador um monitoramento de rotina para o diagnostico e tratamento de maneira oportuna, evitando a sua cronicidade.

PALABRAS-CHAVE: Chlamydia trachomatis, infertilidade. 
INTRODUCCIÓN

En la última década la infección por la endobacteria Chlamydia trachomatis se ha convertido en la enfermedad de transmisión sexual (ETS) más frecuente en todo el mundo ${ }^{1-4}$, especialmente entre adultos jóvenes. ${ }^{5}$

En las mujeres, la bacteria asciende por el tracto reproductor femenino y se puede ocasionar con uretritis, cervicitis, bartolinitis, sangrado uterino irregular, salpingitis y enfermedad inflamatoria pélvica. ${ }^{1-3,6,-9}$ Este microorganismo es responsable de provocar daños en el endometrio, en el tejido fetal ${ }^{10-12}$ y en los cilios de las trompas de Falopio, ocasionando la obstrucción de las mismas, ${ }^{1,7,13}$ lo que aumenta el riesgo de embarazos ectópicos, abortos espontáneos e infertilidad. ${ }^{1-3,5,10,17,18}$ También aumenta el riesgo de adquirir infecciones por virus de inmunodeficiencia humana $(\mathrm{VIH})^{15} \mathrm{y}$ por virus del papiloma humano (VPH).,16,17

En el hombre puede provocar epididimitis, prostatitis, alteraciones en la estructura espermática, así como la formación de anticuerpos antiespermatozoides, lo que puede ser causa de infertilidad masculina. ${ }^{1,4}$

En un alto porcentaje de los casos, la infección cursa de manera asintomática. $3,5,6,18,19$ Cerca de un $70-75 \%$ de las mujeres ${ }^{1,3,16}$ y un $50 \%$ de los hombres no reportan ningún tipo de síntoma mientras tienen la infección, ${ }^{1}$ así puede pasar desapercibida y convertirse en crónica por más de 20 años, ${ }^{1,2}$ transformándose en un serio problema para muchas mujeres, ya que a partir de los 35 años de edad la posibilidad de quedar embarazada disminuye de manera considerable. ${ }^{20,21}$

Existen varios factores de riesgo para adquirir la infección por C. trachomatis, entre los que están: personas con edades comprendidas entre los 15 y 24 años, ser una persona soltera sexualmente activa, tener múltiples compañeros sexuales, usar anticonceptivos orales o dispositivos intrauterinos, tener historia previa de enfermedad de transmisión sexual (ETS), flujo vaginal y dolor abdominal.,16,22

Por este motivo el presente trabajo tiene como objetivo determinar la frecuencia de infección por $C$. trachomatis en mujeres infértiles y su asociación a los diferentes tipos de infertilidad en las mujeres ecuatorianas en edad reproductiva. Como objetivos secundarios, se intentará demostrar la asociación de dicha infección con el tipo de infertilidad (primaria o secundaria), abortos previos, pronóstico reproductivo y las alteraciones en el espermatograma de la pareja sexual de la mujer.

\section{MATERIALES Y MÉTODOS}

Se realizó un estudio transversal donde se revisaron las historias clínicas de las mujeres que acudieron al servicio de Ginecología del hospital Teodoro Maldonado Carbo de la ciudad de Guayaquil, por infertilidad primaria o secundaria desde el 1 de enero de 2012 hasta el 31 de diciembre de 2013.

Los criterios de inclusión fueron: pacientes de sexo femenino que acudieron a la consulta ginecológica por deseos de fertilidad, comprendidas entre los 18 y 50 años de edad, y mujeres que hayan tenido registrado el resultado del test de C. trachomatis. Los criterios de exclusión fueron: pacientes que no presentaron todos los datos requeridos para el estudio.

Se evaluó a 101 pacientes con diagnóstico de infertilidad, que tenían registrado el resultado del test de $\mathrm{C}$. trachomatis.

El test utilizado fue la detección de anticuerpos Ig $M$ e Ig $\mathrm{C}$ contra $\mathrm{C}$. trachomatis por ensayo por inmunoabsorción ligado a enzimas (ELISA).

Mediante las historias clínicas se registraron la edad de las pacientes, el resultado del test de C. trachomatis, el tipo de infertilidad (primaria o secundaria), el antecedente de abortos, las alteraciones en el semen de la pareja sexual de la mujer y el pronóstico reproductivo (bueno o malo) mediante hallazgos laparoscópicos e histerosalpingografía.

En este estudio, las pacientes con mal pronóstico reproductivo eran aquellas que únicamente podían embarazarse por medio de fertilización in vitro.

Los datos fueron recogidos en una hoja de Microsoft Office Excel $2007^{\circledR}$ y para calcular el valor de " $p$ " de las variables cualitativas se utilizó Chi cuadrado en el programa estadístico STATA versión $11.1^{\circledR}$ 
RESULTADOS

De las 101 pacientes que formaron parte de este estudio, $66(65,35 \%)$ tuvieron el test positivo para C. trachomatis. (Tabla 1) y $35(34,65 \%)$ el test negativo. Separando a las mujeres por grupos de edad de 18 a 34 años y de 35 a 50 años, el grupo de edad que mostró una mayor frecuencia de esta infección fue el segundo con un $67,80 \%$ versus un $61,90 \%$ del primer grupo. (Tabla 1). La mayoría de las pacientes con test de $C$. trachomatis positivo tienen entre $36 \mathrm{y}$ 39 años. (Figura 1). El promedio de edad de las pacientes que acudieron por infertilidad fue de 35,85 años.

TABLA 1. PREVALENCIA DE INFECCIÓN POR C. TRACHOMATIS EN MUJERES INFÉRTILES POR GRUPOS DE EDAD

\begin{tabular}{lrrr}
\hline GRUPOS DE EDAD & $\begin{array}{r}\text { MUJERES CON } \\
\text { INFERTILIDAD }\end{array}$ & $\begin{array}{r}\text { MUJERES CON TEST DE } \\
\text { CHLAMYDIA POSITIVO }\end{array}$ & PREVALENCIA \\
\hline $18-34$ & 42 & 26 & $61,90 \%$ \\
\hline $35-50$ & 59 & 40 & $67,80 \%$ \\
\hline Total & 101 & 66 & $65,35 \%$ \\
\hline
\end{tabular}

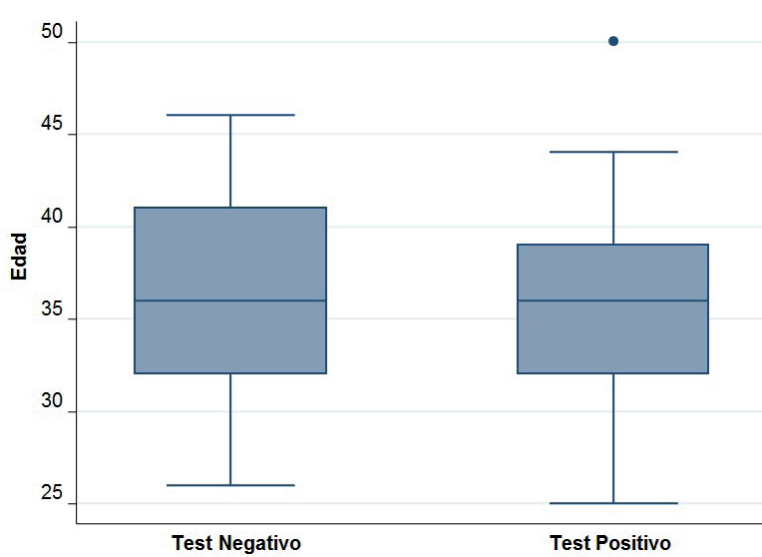

Figura 1. Se puede observar que gran parte de las pacientes con test de C. trachomatis positivo tienen entre 36 y los 39 años.

De las 66 pacientes que acudieron con diagnóstico de infertilidad y que tuvieron un test de C. trachomatis positivo, $30(45,45 \%)$ tenían infertilidad primaria y $36(54,54 \%)$ infertilidad secundaria ( $\mathrm{p}=0,099)$, (Tabla 2); $26(39,39 \%)$ tuvieron abortos y $40(60,61 \%)$ no los tuvieron ( $\mathrm{p}=0,539)$, (Tabla 2); $47(71,21 \%)$ tuvieron mal pronóstico reproductivo y $19(28,79 \%)$ tuvieron buen pronóstico ( $\mathrm{p}=0,089)$, (tabla 2); y $63(95,45 \%)$ tuvieron parejas sexuales con alteraciones en el espermatograma y $3(4,55 \%)$ tuvieron parejas sexuales con un espermatograma normal ( $\mathrm{p}<$ 0,001). (Tabla 2).
TABLA 2. ASOCIACIÓN ENTRE EL RESULTADO DEL TEST DE C. TRACHOMATIS CON: TIPO DE INFERTILIDAD, ABORTOS PREVIOS, PRONÓSTICO REPRODUCTIVO Y ALTERACIONES DEL ESPERMATOGRAMA DE LA PAREJA.

\begin{tabular}{|c|c|c|c|c|}
\hline & $\begin{array}{r}\text { TEST DE } \\
\text { CHLAMYDIA } \\
\text { NEGATIVO }\end{array}$ & $\begin{array}{r}\text { TEST DE } \\
\text { CHLAMYDIA } \\
\text { POSITIVO }\end{array}$ & TOTAL & VALOR DE P \\
\hline Inf. primaria & $\begin{array}{r}10 \\
(28,57 \%)\end{array}$ & $\begin{array}{r}30 \\
(45,45 \%)\end{array}$ & $\begin{array}{r}40 \\
(39,60 \%)\end{array}$ & 0,099 \\
\hline $\begin{array}{l}\text { Inf. } \\
\text { secundaria }\end{array}$ & $\begin{array}{r}25 \\
(71,43 \%)\end{array}$ & $\begin{array}{r}36 \\
(54,55 \%)\end{array}$ & $\begin{array}{r}61 \\
(60,40 \%)\end{array}$ & \\
\hline Total & $\begin{array}{r}35 \\
(100 \%)\end{array}$ & $\begin{array}{r}66 \\
(100 \%)\end{array}$ & $\begin{array}{r}101 \\
(100 \%)\end{array}$ & \\
\hline $\begin{array}{l}\text { No App } \\
\text { aborto }\end{array}$ & $\begin{array}{r}19 \\
(54,29 \%)\end{array}$ & $\begin{array}{r}40 \\
(60,61 \%)\end{array}$ & $\begin{array}{r}59 \\
(58,42 \%)\end{array}$ & 0,539 \\
\hline APP aborto & $\begin{array}{r}16 \\
(45,71 \%)\end{array}$ & $\begin{array}{r}26 \\
(39,39 \%)\end{array}$ & $\begin{array}{r}42 \\
(41,58 \%)\end{array}$ & \\
\hline Total & $\begin{array}{r}35 \\
(100 \%)\end{array}$ & $\begin{array}{r}66 \\
(100 \%)\end{array}$ & $\begin{array}{r}101 \\
(100 \%)\end{array}$ & \\
\hline $\begin{array}{l}\text { P. reproduc- } \\
\text { tivo bueno }\end{array}$ & $\begin{array}{r}16 \\
(45,71 \%)\end{array}$ & $\begin{array}{r}19 \\
(28,79 \%)\end{array}$ & $\begin{array}{r}35 \\
(34,65 \%)\end{array}$ & 0,089 \\
\hline $\begin{array}{l}\text { P. reproduc- } \\
\text { tivo malo }\end{array}$ & $\begin{array}{r}19 \\
(54,29 \%)\end{array}$ & $\begin{array}{r}47 \\
(71,21 \%)\end{array}$ & $\begin{array}{r}66 \\
(65,35 \%)\end{array}$ & \\
\hline Total & $\begin{array}{r}35 \\
(100 \%)\end{array}$ & $\begin{array}{r}66 \\
(100 \%)\end{array}$ & $\begin{array}{r}101 \\
(100 \%)\end{array}$ & \\
\hline $\begin{array}{l}\text { Esperma- } \\
\text { tograma } \\
\text { normal }\end{array}$ & $\begin{array}{r}20 \\
(57,14 \%)\end{array}$ & $\begin{array}{r}3 \\
(4,55 \%)\end{array}$ & $\begin{array}{r}23 \\
(22,77 \%)\end{array}$ & 0,000 \\
\hline $\begin{array}{l}\text { Esperma- } \\
\text { tograma } \\
\text { alterado }\end{array}$ & $\begin{array}{r}15 \\
(42,86 \%)\end{array}$ & $\begin{array}{r}63 \\
(95,45 \%)\end{array}$ & $\begin{array}{r}78 \\
(77,23 \%)\end{array}$ & \\
\hline Total & $\begin{array}{r}35 \\
(100 \%)\end{array}$ & $\begin{array}{r}66 \\
(100 \%)\end{array}$ & $\begin{array}{r}101 \\
(100 \%)\end{array}$ & \\
\hline
\end{tabular}

\section{DISCUSIÓN}

La frecuencia de la infección por C. trachomatis en las pacientes que acudieron por infertilidad al servicio de ginecología del hospital Teodoro Maldonado Carbo, desde el 1 de enero de 2012 hasta el 31 de diciembre de 2013, fue de $65,25 \%$, lo que indica que esta infección es común entre las mujeres infértiles de nuestro medio.

El hecho de que el grupo de 35 a 50 años presentara una mayor prevalencia $(67,80 \%)$ de infección por C. trachomatis que el grupo de 18 a 24 años $(61,90 \%$ y de que la mayoría de las pacientes con esta infección tengan entre 36 y 39 años, disminuye aun más la fertilidad en estas mujeres, ya que en este caso no solo influye estar infectada por esta bacteria, sino también que por estar encima de los 35 años de edad, aumentan los factores para la infertilidad. ${ }^{20,21}$ 
Al evaluar la asociación entre las pacientes que tuvieron un test positivo, que fueron $66(65,35 \%)$, con el tipo de infertilidad, el antecedente de abortos, el pronóstico reproductivo y las alteraciones en el espermatograma de su pareja sexual, sólo se observó una importancia estadísticamente significativa con la alteración del espermatograma $(\mathrm{p}<0,001)$, ya que $63(95,45 \%)$ de las parejas tenían un espermatograma alterado. Este hecho puede aumentar el riesgo de infertilidad, pero por factor masculino, ya que la infección por C. trachomatis puede producir daño severo en la estructura espermática y a la formación de anticuerpos antiespermatozoides.

En cuanto al antecedente de abortos, no hubo una asociación estadísticamente significativa $(\mathrm{p}=0,539)$, pero probablemente se deba a que una gran parte de la muestra estudiada estaba compuesta por mujeres con infertilidad primaria (40 de 101 que equivale al 39,60\%). Una mujer con infertilidad primaria es aquella que nunca ha quedado embarazada después de al menos un año de tener relaciones sexuales sin protección. A diferencia de una mujer con infertilidad secundaria, que es aquella que ha estado embarazada en al menos una ocasión, pero no ha podido embarazarse nuevamente. ${ }^{23}$ Por este motivo se cree no haber encontrado una asociación estadísticamente significativa con esta variable. Para poder descartar esta asociación se debería realizar una investigación en pacientes con infertilidad secundaria, ya que al haber quedado al menos una vez embarazadas, tienen la probabilidad de tener antecedentes de abortos previos.

De las 66 pacientes con test de chlamydia positivo, 47 (77,21\%) tuvieron mal pronóstico reproductivo, diagnosticado mediante hallazgos laparoscópicos e histerosalpingografía. Esta cifra es muy alta y preocupante, ya que al tener un pronóstico reproductivo malo, estas pacientes probablemente no logren quedar embarazadas en el futuro, a menos que se realicen técnicas de reproducción asistida avanzadas, procedimientos que no cubre el Instituto Ecuatoriano de Seguridad Social, y realizarlas de manera privada tiene un costo muy elevado.

Pese a que las pacientes antes mencionadas tuvieron una prevalencia alta de infección por C. trachomatis, la asociación no tuvo significancia estadística $(\mathrm{p}=0,089)$, probablemente por el tamaño de la muestra del estudio (101 pacientes), que fue muy pequeña, sin embargo existe una tendencia importante a relacionar esta variable con la infección por $\mathrm{C}$. trachomatis; $\mathrm{y}$ se cree que si se realiza otro estudio similar, con un mayor número de pacientes, probablemente se encuentre una asociación estadísticamente significativa.

Una de las desventajas del estudio es que el tamaño de la muestra fue muy pequeño y esta puede ser la causa de no encontrar diferencia estadísticamente significativa entre la asociación de la infección por $C$. trachomatis con las variables estudiadas, con la excepción de la alteración en el espermatograma de las parejas sexuales de las mujeres. La principal limitación para este estudio se debió a que el registro estadístico del hospital Teodoro Maldonado Carbo no cuenta con un mayor número de datos de pacientes evaluadas en el tiempo establecido.

\section{CONCLUSIONES}

La prevalencia de la infección por C. trachomatis en mujeres infértiles que acudieron a la consulta ginecológica en el hospital Teodoro Maldonado Carbo es alta, lo cual es consistente con varios estudios realizados en distintos países. ${ }^{1-5,13}$

Se recomienda implementar en el hospital Teodoro Maldonado Carbo un examen rutinario de C. trachomatis o realizar futuros estudios que lo justifiquen, como lo aconsejan Urbina et $\mathrm{al}^{1}$, Preciado Ruiz et $\mathrm{al},{ }^{4} \mathrm{Kl}$ øvstad et al, ${ }^{24}$ y Van Den Broek et al, ${ }^{25}$ incluyendo su determinación en controles ginecológicos anuales, así como evaluaciones urológicas a hombres jóvenes, para de esta manera diagnosticar, tratar y erradicar la infección mediante un seguimiento.

Se espera que esta recomendación sea acogida en los demás centros de salud que también cuenten con prevalencia elevada de esta infección. Con esta medida se desea disminuir la prevalencia de la infección y todas las complicaciones que se producen en el tracto reproductor femenino. Es importante insistir en que el tratamiento debe ser para la mujer y su pareja. También se recomienda que los profesionales de la salud colaboren en la difusión de los riesgos de esta infección y las consecuencias de la misma, sobre todo a jóvenes y adolescentes, así como lo realizan con otras ETS como el VIH, VPH, entre otras. 


\section{REFERENCIAS BIBLIOGRÁFICAS}

1. Urbina MT, Medina R, Muñoz G, Sánchez V, Benjamín, Lerner J. Infección por Chlamydia trachomatis. Revista de Obstetricia y Ginecología de Venezuela. 2010 June; 70(2):90 96.

2. Jeremiah I, Okike O, Akani C. The Prevalence of Serum Immunoglobulin C Antibody to Chlamydia Trachomatis in Subfertile Women Presenting at the University of Port Harcourt Teaching Hospital, Nigeria. International Journal Of Biomedical Science. 2011; 7(2): 120 - 124.

3. Siemer J, Theile O, Larbi Y, Fasching P, Danso K, Kreienberg $\mathrm{R}$, et al. Chlamydia trachomatis Infection as a Risk Factor for Infertility among Women in Chana, West Africa. American Journal of Tropical Medicine and Hygene. 2008; 78(2): 323-327.

4. Preciado R, Arredondo R, García A, Manterola D, Blanco $\mathrm{N}$, Martínez J. Indentificación de Chlamydia trachomatis en parejas infértiles. Revista Mexicana de Medicina de la Reproducción. 2011 Octubre - Diciembre; 4(2): 72 -76.

5. Surana A, Rastogi V, Singh Nirwan P. Association of the Serum Anti-chlamydial Antibodies with Tubal Infertility. Journal of Clinical and Diagnostic Research. 2012 December; 6(10): 1692-1694.

6. Kohli R, Konya W, Obura T, Stones W, Revathi G. Prevalence of genital chlamydia infection in urban women of reproductive age, Nairobi, Kenya. BMC Research Notes 2013 February; 6: 44.

7. Hjelholt A, Christiansen G, Gravesen T, Jakob H, Birkelund S. Tubal factor infertility is associated with antibodies against Chlamydia trachomatis heat shock protein 60 (HSPGo) but not human HSP6o. Human Reproduction. 2011 June; 26(8): $2069-2067$.

8. Öhman H, Bailey R, Natividad A, Ragoussis J, Johnson L-L, Tiitinen A, et al. Effect of IL12A and IL12B polymorphisms on the risk of Chlamydia trachomatis-induced tubal factor infertility and disease severity. Human Reproduction. 2012 May; 27(7): 2217-2223.

9. Bakken I, Chaderi S. Incidence of pelvic inflammatory disease in a large cohort of women tested for Chlamydia trachomatis: a historical follow-up study. BMC Infectious Diseases 2009; 9:130.

10. Baud D, Goy G, Jaton K, Osterheld M-C, Blumer S, Borel N, et al. Role of Chlamydia trachomatis in Miscarriage. Emerging Infectous Diseases. 2011 September; 17(9): 1630-1635.

11. Coppus S, Land J, Opmeer B, Steures P, Eijkemans M, Hompes P, et al. Chlamydia trachomatis IgC seropositivity is associated with lower natural conception rates in ovulatory subfertile women without visible tubal pathology. Human Reproduction. 2011 September; 26(11): 3061-3067.

12. Bilardi J, De Guingand D, Temple-Smith M, Garland S, Fairley C, Grover S, et al. Young pregnant women's views on the acceptability of screening for chlamydia as part of routine antenatal care. BMC Infectious Diseases 2013, 13:30.

13. Ortiz C, Hechavarría C, Ley M, Álvarez G, Hernández Y. Estudio de Chlamydia trachomatis, Ureaplasma urealyticum y Mycoplasma hominis en pacientes infértiles y abortadoras habituales. Revista Cubana de Obstetricia y Ginecología.2010; 36(4): 573-584.
14. Casari E, Ferrario A, Morenghi E, Montanelli A. Gardnerella, Trichomonas vaginalis, Candida, Chlamydia trachomatis, Mycoplasma hominis and Ureaplasma urealyticum in the genital discharge of symptomatic fertile and asymptomatic infertile women. New Microbiologica. 2010; 33: 69-76.

15. Beydoun H, Dail J, Tamim H, Ugwu B, Beydoun M. Gender and Age Disparities in the Prevalence of Chlamydia Infection Among Sexually Active Adults in the United States. Journal Of Women's Health. 2010; 19(12): 2183-2190.

16. Farinati A, Zitto T, Bottiglieri M, Gastaldello R, Cuffini C, Cannistraci R, etal. Infecciones asintomáticas por Chlamydia trachomatis: un problema controlable en la población adolescente. Revista Panamericana de Infectología. 2008; $10(1): 8-12$.

17. Hernández M, López M, Arteaga G, Guerra F. Evaluación de los niveles séricos de anticuerpos IgC anti-Chlamydia trachomatis para el diagnóstico de infertilidad por factor tubario. Revista de Investigación Clínica. 2009 Enero Febrero; 61(1): 87-89.

18. Singh D, Fine D, Marrazzo J. Chlamydia trachomatis InfectionAmongWomenReporting Sexual Activity With Women Screened in Family Planning Clinics in the Pacific Northwest, 1997 to 2005. American Journal of Public Health. 2011 July; 101(7): 1284-1290.

19. Herzog S, Althaus C, Heijne J, Oakeshott P, Kerry S, Hay P, et al. Timing of progression from Chlamydia trachomatis infection to pelvic inflammatory disease: a mathematical modelling study. BMC Infectious Diseases 2012; 12:187.

20. Pfeifer S, Goldberg J, Lobo R, Thomas M, Pisarska M, Widra E, et al. Optimizing natural fertility: a committee opinión. Fertility and Sterility. 2013 September; 100(3): 631-637.

21. Lampinen R, Vehviläinen-Julkunen K, Kankkunen P. A Review of Pregnancy in Women Over 35 Years of Age. The Open Nursing Journal. 2009; 3: 33-38.

22. Kløvstad H, Griibovski A, Aavitsland P. Population based study of genital Chlamydia trachomatis prevalence and associated factors in Norway: A cross sectional study. BMC Infectious Diseases 2012, 12:150.

23. Schorge J, Schaffer J, Halvorson L, Hoffman B, Bradshaw $\mathrm{K}$, Cunningham F, et al. Valoracion de la pareja estéril. En: Schorge J, Schaffer J, Halvorson L, Hoffman B, Bradshaw K, Cunningham F. Williams Cinecología. 1a edición. México; McCraw Hill; 2009. p. 426 - 446.

24. Kløvstad H, Natås O, Tverdal A, Aavitsland P. Systematic screening with information and home sampling for genital Chlamydia trachomatis infections in young men and women in Norway: a randomized controlled trial. BMC Infectious Diseases 2013; 13:30.

25. Van Den Broek I, Van Bergen J, Brouwers E, Fennema J, Götz $\mathrm{H}$, Hoebe $\mathrm{C}$, et al. Effectiveness of yearly, register based screening for chlamydia in the Netherlands: controlled trial with randomised stepped wedge implementation. British Medical Journal. 2012; 345:4316. 